\title{
Characterization of new ferromagnetic Fe-Co-Zn-Ga alloys by ab initio investigations
}

\author{
Antje Dannenberg ${ }^{1, a}$, Markus E. Gruner ${ }^{1}$, Manfred Wuttig $^{2}$, and Peter Entel ${ }^{1}$ \\ 1 Department of Physics and Center for Nanointegration, CENIDE, University of Duisburg-Essen, \\ 47048 Duisburg, Germany \\ 2 Department of Materials Science and Engineering, University of Maryland, College Park, MD 20742- \\ 2115 , USA
}

\begin{abstract}
We have calculated structural energy differences, magnetic interaction constants and mixing energies of $\mathrm{Fe}-\mathrm{Co}-\mathrm{Ga}(\mathrm{Zn})$ alloys in order to gain information about their suitability for ferromagnetic shape memory devices. We considered the classical Heusler structure as well as an ordering type in which the Co and one Fe sublattice are interchanged. The results of our density functional theory investigations suggest high Curie and martensitic phase transition temperatures for the Ga-based as well as for the Zn-based systems. In case of hypothetical $\mathrm{Fe}_{2} \mathrm{CoZn}$ alloys, the classically ordered Heusler structure is energetically preferred. In Ga based alloys, the ordering type with Co partially on Fe sites appears to be more stable. We propose that a systematic variation of composition by successive addition of $\mathrm{Zn}$ and $\mathrm{Co}$ to $\mathrm{Fe}-\mathrm{Co}-\mathrm{Ga}$ may result in a promising new ferromagnetic shape memory alloy of type $\mathrm{Fe}_{2-\mathrm{x}} \mathrm{Co}_{1+\mathrm{x}} \mathrm{Ga}_{1-\mathrm{y}} \mathrm{Zn}_{\mathrm{y}}$.
\end{abstract}

\section{Introduction}

Ferromagnetic shape memory alloys (FSMAs) have attracted considerable attention due to their potential use for new actuator materials. For prototype $\mathrm{Ni}_{2} \mathrm{MnGa}$ Heusler compound, large magnetically induced strains have been reported in the martensitic phase. However, the martensitic phase transition takes place at too low temperatures for many practical applications. Furthermore, Ni-Mn-Ga alloys are disadvantageous because of their poor room temperature ductility or high costs of elements [1-3]. In the literature, various other systems including Ni-Mn-(Al, Sn, $\mathrm{Sb}, \mathrm{In})[4,5], \mathrm{Co}-\mathrm{Ni}-(\mathrm{Al}, \mathrm{Ga})[6-13], \mathrm{Ni}-\mathrm{Fe}-(\mathrm{Al}, \mathrm{Ga})[14]$ and $\mathrm{Cu}-\mathrm{Mn}-(\mathrm{Al}, \mathrm{Ga})[15,16]$ have been proposed as candidates for new FSMAs. Of special interest are Heusler systems based on Co, $\mathrm{Fe}$, and $\mathrm{Zn}$ : For the $\mathrm{Co}-\mathrm{Ni}-(\mathrm{Al}, \mathrm{Ga})$ and Ni-Ga-Fe-Co alloys, the martensitic start temperature $\mathrm{T}_{S}$ decreases with increasing Co content while Curie temperature and spontaneous magnetization increase $[17,18]$. For Ni-Mn-Ga alloys, the martensitic transformation temperature $\mathrm{T}_{M}$ can be increased by changing the stoichiometry. Recently developed ferromagnetic Ni-Fe-Ga alloys show good plasticity of the high-temperature phase in comparision to $\mathrm{Ni}_{2} \mathrm{MnGa}$ and thus may be promising. In Fe-Co-Zn, the magnetic phase transition induces various interesting anomalies like a miscibility gap between the ferromagnetic and the paramagnetic bcc $\alpha$-phase [19]. The nonmagnetic $\mathrm{Cu}-\mathrm{Al}-\mathrm{Zn}$ Heusler alloys are important SMA [20,21] while Fe-Ga is a magnetostrictive material $[22,23]$. The compound $\mathrm{Co}_{2} \mathrm{FeGa}$ exhibits the classically ordered Heusler structure as early experimental studies report [24], while in Fe-Co-Ga, the Co atoms prefer to occupy parts of the Fe sublattices (e.g., the B site, see Fig. 1). Thus, one has to differentiate two types of Fe atoms: Fe atoms which remain on the Fe sublattice (e.g., the A site, here denoted

\footnotetext{
a e-mail: antje@thp.uni-duisburg.de
} 


\section{$\mathrm{Fe}_{2} \mathrm{CoGa}(\mathrm{Zn})$}

(a) $\mathrm{ABCD}$

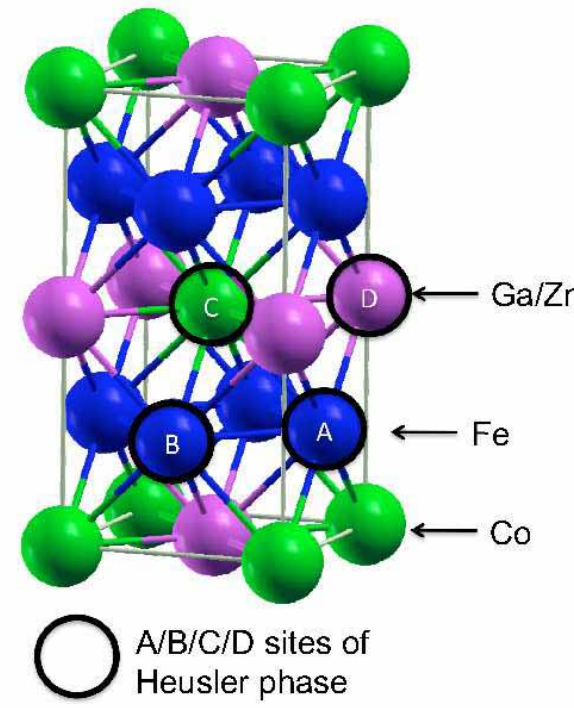

(b) ACBD

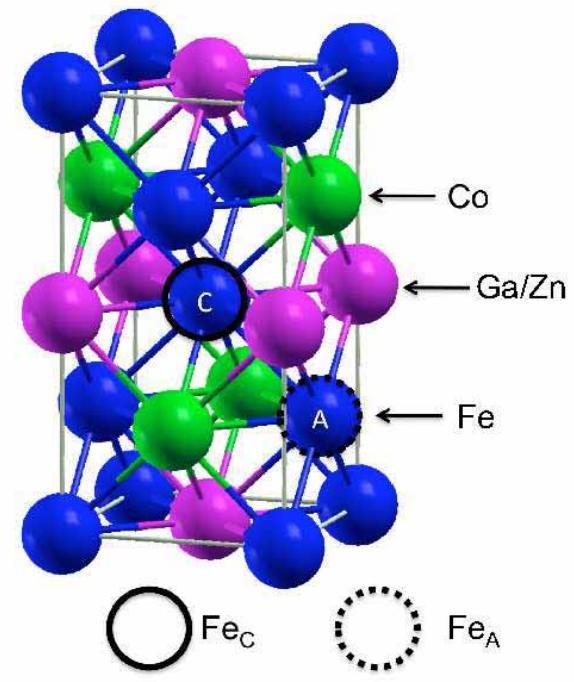

Fig. 1. Left: Tetragonal, body-centered unit cell in the $\mathrm{Fe}_{2} \mathrm{CoGa}(\mathrm{Zn})$ Heusler structure, named $\mathrm{ABCD}$ in the order of appearance in the chemical formula. Right: Heusler unit cell with Co atoms (green spheres) on one Fe (blue spheres) sublattice, marked as ACBD. Here, half of the Fe atoms (B sites) occupy the Co sites ( $\mathrm{C}$ sites and thus are named as $\mathrm{Fe}_{\mathrm{C}}$ ) while the remaining half of the Fe atoms stay at their sites, and are consequently denoted by $\mathrm{Fe}_{\mathrm{A}} \cdot \mathrm{Ga}(\mathrm{Zn})$ atoms are shown as magenta spheres.

by $\mathrm{Fe}_{A}$ ) and $\mathrm{Fe}$ atoms which occupy Co sites (the $\mathrm{C}$ site, denoted by $\mathrm{Fe}_{C}$ ) (see Fig. 1). At $25 \% \mathrm{Co}$, ordering type ACBD, with the elements placed on the sites in the respective order of their appearance in the formula unit (cf. Fig. 1), is achieved and with further increasing Co content the Fe sublattices becomes entirely occupied by Co atoms, so that we obtain the order $\mathrm{ABCD}$ for $\mathrm{Co}_{2} \mathrm{FeGa}$. In general, transition metal atoms to the right of $\mathrm{Fe}$ in the periodic table preferentially go to the Fe sublattice (e.g B site) while those to the left of Fe go to the Co sites (e.g. C site) [24]. A considerable Curie temperature of the order of $1160 \mathrm{~K}$ is found for $\mathrm{Fe}_{2} \mathrm{CoGa}$.

These findings motivate a theoretical investigation of $\mathrm{Fe}-\mathrm{Co}-\mathrm{Ga}(\mathrm{Zn})$ alloys in the classical Heusler structure (ABCD) and in the ordering type ACBD (see Fig. 1). Quaternary alloys of type Fe-Ni-Co-Ga-Zn may be promising in order to increase the range of potential appliction of FSMAs and for an appropriate choice of composition, high transition temperatures and an improvement of mechanical properties may be obtained.

\section{Computational details}

Our investigations were carried out in the framework of density functional theory using the plane wave code VASP [25]. The electron-ionic core interaction is accounted for by applying the PAW method [26] and the GGA exchange-correlation potential of Perdew, Burke, and Ernzerhoff [27]. The integration over the Brillouin zone was carried out using the tetrahedron method with Blöchel corrections and $\Gamma$-centered k-point grids of $13 \times 13 \times 13$ (total energy) and 
Table 1. Calculated results of tetragonally distorted Fe-Co-Ga( $\mathrm{Zn}), c / a \neq 1$, optimum lattice constant, $a$, magnetic moment, $\mathrm{M}$, mixing energy, $\mathrm{E}_{\text {mix }}$, energy difference between the energy minimum at $c / a<1$ and the energy minimum at $c / a>1, \Delta \mathrm{E}_{\mathrm{c} / \mathrm{a}}$, and corresponding approximated martensitic phase transition temperature, $\mathrm{T}_{M}=\Delta \mathrm{E}_{\mathrm{c} / \mathrm{a}}$ in $\mathrm{K}$, nearest neighbor magnetic interaction constant, $\mathrm{J}_{\mathrm{ij}}$, with resulting mean-field Curie temperature $\mathrm{T}_{\mathrm{C}}$, and the energy difference between the $\mathrm{ABCD}$ and the ACBD order (see Fig. 1), $\Delta \mathrm{E}_{\text {order }}$.

\begin{tabular}{|c|c|c|c|c|c|c|c|c|}
\hline \multirow[b]{3}{*}{$\mathrm{c} / \mathrm{a}$} & & \multicolumn{4}{|c|}{$\mathrm{Fe}_{2} \mathrm{CoZn}$} & \multicolumn{3}{|c|}{$\mathrm{Fe}_{2} \mathrm{CoGa}$} \\
\hline & & \multicolumn{2}{|c|}{$\mathrm{ABCD}$} & \multicolumn{2}{|c|}{$\mathrm{ACBD}$} & \multicolumn{2}{|c|}{$\mathrm{ABCD}$} & \multirow{2}{*}{$\begin{array}{c}\mathrm{ACBD} \\
1.0 \\
\end{array}$} \\
\hline & & 0.92 & 1.4 & 1.0 & 1.36 & 0.88 & 1.46 & \\
\hline $\mathrm{a}$ & $(\AA)$ & \multicolumn{2}{|c|}{5.782} & \multicolumn{2}{|c|}{5.750} & \multicolumn{2}{|c|}{5.774} & 5.736 \\
\hline $\mathrm{M}$ & ( $\mu_{\mathrm{B}} /$ f.u. $)$ & 6.65 & 6.64 & 5.95 & 6.24 & 6.07 & 6.08 & 5.29 \\
\hline $\mathrm{E}_{\text {mix }}$ & (meV/atom) & 232 & 51 & 92 & 135 & -257 & -463 & -642 \\
\hline$\Delta \mathrm{E}_{\mathrm{c} / \mathrm{a}}$ & (meV/atom) & 44 & & -11 & & 52 & & \\
\hline $\mathrm{T}_{M}$ & $(\mathrm{~K})$ & 483 & & & & 563 & & \\
\hline $\mathrm{J}_{\mathrm{ij}}$ & (meV/atom) & 23 & 20 & 19 & & 18 & 14 & 23 \\
\hline $\mathrm{T}_{C}$ & $(\mathrm{~K})$ & 1930 & 1725 & 1716 & & 1540 & 1232 & 1645 \\
\hline$\Delta \mathrm{E}_{\text {order }}$ & (meV/atom) & \multicolumn{4}{|c|}{-8.89} & \multicolumn{3}{|c|}{44.42} \\
\hline
\end{tabular}

$19 \times 19 \times 19$ (mixing energies). The energy cut-off (in eV) was chosen as 366.5 for $\mathrm{Fe}_{2} \mathrm{CoGa}(\mathrm{Zn})$ and $\mathrm{Co}_{2} \mathrm{FeZn}$.

The mixing energies are calculated using the simple formula: $\Delta E_{\operatorname{mix}}=\mathrm{E}_{\text {alloy }}-\sum_{i} c_{i} E_{i}$. Here $E_{\text {alloy }}$ is the ground state energy of the respective alloy in $\mathrm{eV} / \mathrm{f}$.u. and $\mathrm{E}_{i}$ is the bulk energy in $\mathrm{eV} /$ atom of material component $i$, and $c_{i}$ their respective weight in the formula unit. For the determination of the magnetic exchange interaction constants $\mathrm{J}_{\mathrm{ij}}$, we applied the Munich spin polarized relativistic Korringa-Kohn-Rostoker (SPR-KKR) package, version 3.6 [28,29]. The angular momentum expansion was truncated after the $1=3$ component. In the classical Heusler structure (order type ABCD, see Fig. 1) a k-point mesh of $22 \times 22 \times 22$ and for the ACBD order a mesh of $12 \times 12 \times 12$ was used. The Wigner-Seitz radii (in $\AA$ ) are choosen for $\mathrm{Fe}_{2} \mathrm{CoZn}$ in ABCD order as: $\mathrm{Fe}=2.669, \mathrm{Co}=2.628$, and $\mathrm{Zn}=2.787$ and for $\mathrm{ACBD}$ order as: $\mathrm{Fe}=2.654, \mathrm{Co}=2.613$, and $\mathrm{Zn}=2.772$. For $\mathrm{Fe}_{2} \mathrm{CoGa}$ in $\mathrm{ABCD}$ order we get: $\mathrm{Fe}=2.673$, Co $=2.632$, and $\mathrm{Ga}=2.761$ and in $\mathrm{ACBD}$ order we used $\mathrm{Fe}=2.655, \mathrm{Co}=2.615$, and $\mathrm{Ga}=2.743$.

\section{Results and discussion}

In order to investigate the structural stability of Fe-Co-Ga(Zn) alloys, we calculated the mixing energy, $\mathrm{E}_{\mathrm{mix}}$, and the energy difference between the classically Heusler order (type ABCD) and FeCoFeGa(Zn) order (type ACBD), $\Delta \mathrm{E}_{\text {order }}$, both in meV/atom (compare Table 1 and Fig. 1). Thus, a negative $\Delta \mathrm{E}_{\text {order }}$ means that the $\mathrm{ABCD}$ order is energetically preferred. When performing a tetragonal c/a distortion of the ABCD structure, two energy minima are observed. The energy difference between these minima, $\Delta \mathrm{E}_{\mathrm{c} / \mathrm{a}}$, expressed in $\mathrm{K}$, gives a rough measure of the martensitic phase transition temperatures $\mathrm{T}_{M}$ (Table 1). For the ordering type ACBD with Co on half of the Fe sites (cf. Fig. 1), the cubic phase with $c / a=1$ is clearly favorable (see Fig. 2) From the calculated magnetic exchange interaction constants (Table 1 and Fig. 3) we evaluated the Curie temperatures using a simple Heisenberg model within mean field approximation: $\mathrm{T}_{\mathrm{C}}=\mathrm{zM}_{\mathrm{i}} \mathrm{M}_{\mathrm{j}} \mathrm{J}_{\mathrm{ij}}$, where $\mathrm{z}$ is the number of nearest neighbors and $M_{i, j}=1$ for ferromagnetic coupling. Here, we find extraordinarily high $\mathrm{T}_{C}$ values in the range of $1232 \mathrm{~K}$ up to $1645 \mathrm{~K}$.

For Fe-Co-Ga the mixing energies are negative (cf. Table 1). And as early experimental studies confirm, these alloys are in fact stable [24]. The calculated $\mathrm{J}_{\mathrm{ij}}$ predict strong ferromagnetism (cf. Fig. 3) and extremly high Curie temperatures of the order of $1232 \mathrm{~K} \leq \mathrm{T}_{C} \leq 1645 \mathrm{~K}$. Also, for the case that Fe atoms become nearest neighbors as in order type $\mathrm{ACBD}$, no antiferromagnetic tendency has been observed in the calculation. As is obvious from Fig. 2, the stable state at zero temperature is the cubic structure with order type ACBD in accordance with experiment. 


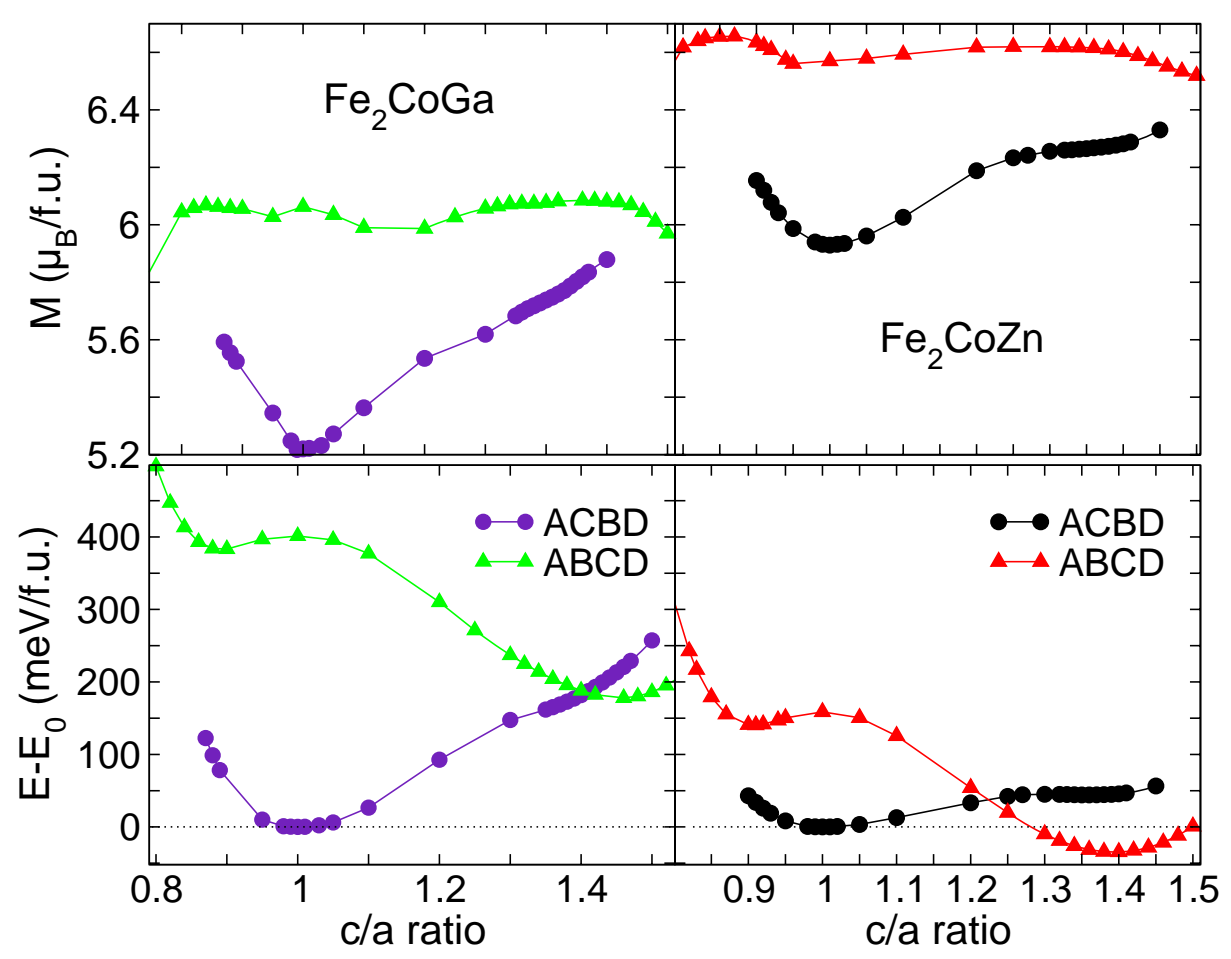

Fig. 2. The variation of the tetragonal distortion, c/a, and the corresponding magnetic moments, $\mathrm{M}$ (in $\mu_{B} /$ f.u.), for Fe-Co-Ga (left) and Fe-Co-Zn (right). Circles mark ordering type ACBD while triangles mark the classical Heusler in ordering type ABCD where Co and $\mathrm{Ga}(\mathrm{Zn})$ share one simple cubic sublattice (cf. Fig. 1). For ordered ABCD, two energy minima are found while for ACBD with Co on part of the Fe sublattice (B site), the cubic phase is clearly favoured.

Therefore, a martensitic phase transition is not likely to be expected in the Ga based alloy. Nevertheless assuming an artificial stabilization of the ABCD phase, we estimate a fairly high hypothetical $\mathrm{T}_{M}=563 \mathrm{~K}$ by expressing $\Delta \mathrm{E}_{\mathrm{c} / \mathrm{a}}$ in $\mathrm{K}$. But at the same time, a phase transition would probably be accompanied with a diffusive rearrangement of Co atoms and thus be not martensitic. In order to confirm the proposed tendency of Co atoms occupying part of the Fe sites (e.g. B site), we performed analogous calculations for $\mathrm{Co}_{2} \mathrm{FeZn}$ (not shown). And indeed, we again find a negative mixing energy but only one energy minimum at $c / a=1$. The magnetic moment per formula unit is of the order $6 \mu_{B}$ for the ABCD order and in between 5.2 and $5.6 \mu_{B}$ for the ACBD structure.

For Fe-Co-Zn, the ABCD structure is found to be more favorable than the ACBD structure and our investigations would predict similar high Curie and martensitic temperatures (1725 K and $483 \mathrm{~K}$, respectively, cf. Table 1). However, the calculated mixing energies appear to be positive for the classical Heusler structure (ABCD) as well as for the ACBD order. This means that at least for the stoichiometric composition, this system is not expected to be stable. But systematic alloying of $\mathrm{Zn}$ to $\mathrm{Fe}-\mathrm{Co}-\mathrm{Ga}$ may stabilize the $\mathrm{ABCD}$ structure and result in an interesting quaternary Fe-Co-Ga-Zn ferromagnetic shape memory alloy with high Curie and martensite temperatures. The magnetic moment per formula unit is enhanced by $8 \%$ in comparision to $\mathrm{Fe}-\mathrm{Co}-\mathrm{Ga}$. This enhancement is due to an increased Fe moment of about $6 \%$ and an increased Co moment of about $11 \%$. In summary, our result show that:

(a) In Fe-Co-Ga the mixing energies are negative for ABCD and ACBD order. An extraordinarily high $\mathrm{T}_{C}$ is predicted $(\sim 1645 \mathrm{~K})$. But here the ACBD order is preferred over the ABCD order. Assuming a stabilisation of ABCD order a high $\mathrm{T}_{M}$ of $563 \mathrm{~K}$ can be expected. Still, not a martensitic transition but a diffusive rearrangement of Co atoms should be expected.

(b) The mixing energies for Fe-Co-Zn are positive. The magnetic moments are enhanced of about 

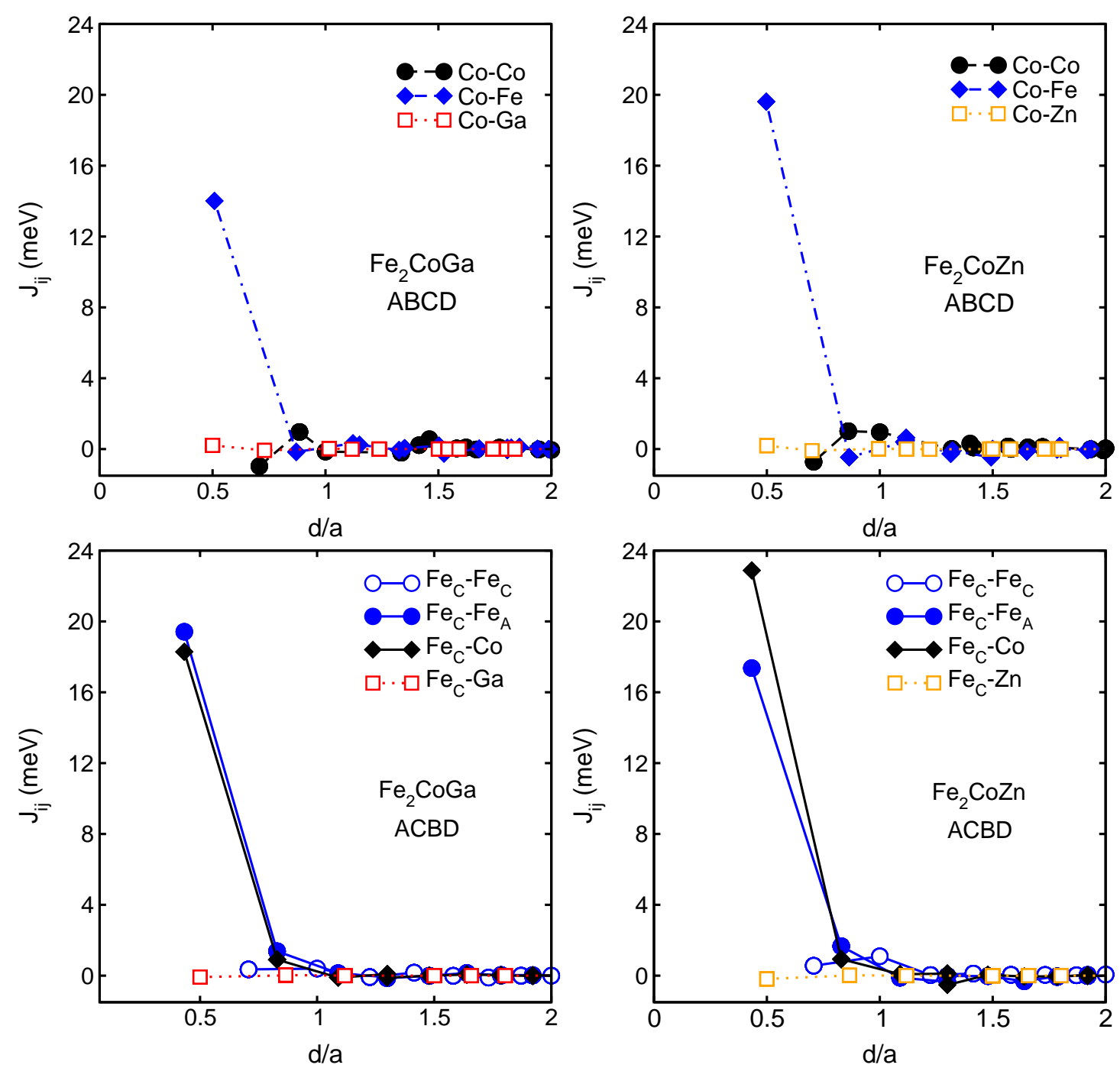

Fig. 3. Magnetic exchange constants $\mathrm{J}_{\mathrm{ij}}$ as a function of the distance $d$ in units of the lattice constant $a$ of $\mathrm{Fe}-\mathrm{Co}-\mathrm{Ga}(\mathrm{Zn})$. The distance is measured from site $\mathrm{C}$ (cf. Fig. 1). Left upper panel: Exchange constants of $\mathrm{Fe}_{2} \mathrm{CoGa}$ in ABCD order at $c / a=1.46$. Left lower panel: $\mathrm{Fe}_{2} \mathrm{CoGa}$ in ACBD order at $c / a=1$. Right upper panel: $\mathrm{Fe}_{2} \mathrm{CoZn}$ in $\mathrm{ABCD}$ order at $c / a=1.4$. Right lower panel: $\mathrm{Fe}_{2} \mathrm{CoZn}$ in $\mathrm{ACBD}$ order at $c / a=1$. For the ABCD structures (upper panels) filled black cirles denote the Co-Co interaction, filled blue diamonds mark the interaction between Co and Fe atoms, and open red (orange) squares belong to $\mathrm{Co}-\mathrm{Ga}(\mathrm{Zn})$ interaction. For the ACBD order (lower panel), open blue circles mark the interaction between $\mathrm{Fe}$ atoms of the same type $\left(\mathrm{Fe}_{\mathrm{C}}-\mathrm{Fe}_{\mathrm{C}}\right)$ while filled blue circles denote the exchange interaction between nearest neighbor $\mathrm{Fe}$ atoms $\left(\mathrm{Fe}_{\mathrm{C}}\right.$ with $\mathrm{Fe}_{\mathrm{A}}$, see Fig. 1). Black filled diamonds belong to interaction of $\mathrm{Fe}_{\mathrm{C}}$ with $\mathrm{Co}$ atoms while red (orange) open squares mark the interaction between $\mathrm{Fe}_{\mathrm{C}}$ atoms and $\mathrm{Ga}(\mathrm{Zn})$ atoms. Strong ferromagnetism is obtained even if Fe atoms are nearest neighbors as for ACBD order.

8\%. For a hypothetical Fe-Co-Zn Heusler alloy, the ABCD order with a tetragonal distorted c/a ratio of 1.4 would be more stable than the cubic ACBD structure. Strong ferromagnetism $\left(\mathrm{T}_{C}=1725 \mathrm{~K}\right)$ and high martensitic phase transition temperatures should be expected. But analogously to Fe-Co-Ga, a diffusive transition with a rearrangement of Co atoms may occur because the ACBD order with cubic symmetry lies only $8.8 \mathrm{meV} /$ atom higher in energy than tetragonally distorted ABCD. 
(c) For the stable $\mathrm{Co}_{2} \mathrm{FeZn}$ system, the mixing energy is negative but only one energy minimum at $c / a=1$ is found.

These findings led us to the tentative conclusion, that, although Fe-Co-Zn is not stable, the addition of $\mathrm{Ga}$ to a hypothetical $\mathrm{Fe}_{2} \mathrm{CoZn}$ Heusler alloy may lead to an interesting quaternary Fe-Co-Zn-Ga FSMA. But attention has to be paid to the possibility of a diffusive transition accompanied with a rearrangement of atoms, due to the fact that at least for stoichiometric $\mathrm{Fe}_{2} \mathrm{CoGa}$, Co atoms are expected to occupy part of the Fe sublattice (e.g., the B sites) and thus hinder the martensitic transformation. Further addition of Co leads again to negative mixing energies. But in the case of $\mathrm{Co}_{2} \mathrm{FeZn}$, the cubic system is the groundstate and no martensitic phase transition is expected. Thus, a careful variation of composition of the quaternary alloy $\mathrm{Fe}_{2-x} \mathrm{Co}_{1+x} \mathrm{Zn}_{1-y} \mathrm{Ga}_{y}$ may result in a new ferromagnetic shape memory alloy with improved properties such as very high Curie and martensite temperature, eventually avoiding brittleness of prototype $\mathrm{Ni}_{2} \mathrm{MnGa}$ Heulser alloy.

\section{Acknowledgment}

Special thanks to Dr. Heike Herper for fruitful and important discussions. Financial support was granted by the Deutsche Forschungsgemeinschaft (SPP 1239).

\section{References}

1. H.E. Karaca, I. Karaman, B. Basaran, Y.I. Chumlyakov, H.J. Maier, Acta Materialia 54, 233 (2006)

2. M.A. Marioni, R.S. O'Handley, S.M. Allen, S.R. Hall, D.I. Paul, M.L. Richard, J. Feuchtwanger, B.W. Peterseon, J.M. Chambers, R. Techapiesancharoenkij, J. Magn. Magn. Mater. 290, 35 (2005)

3. P. Müllner, V.A. Chernenko, G. Kostorz, J. Magn. Magn. Mater. 267, 325 (2003)

4. R. Kainuma, F. Gejima, Y. Sutou, I. Ohnuma, K. Ishida, Mater. Trans. JIM 41, 943 (2000)

5. Y. Sutou, Y. Imano, N. Koeda, T. Omori, R. Kainuma, K. Ishida, K. Oikawa, Appl. Phys. Lett. 85, $4358(2004)$

6. W. Maziarz, J. Dutkiewicz, R. Santamarta, E. Cesari, Eur. Phys. J. Special Topics 158, 137 (2008)

7. Y. Tanaka, K. Oikawa, Y. Sutou, T. Omori, R. Kainuma, K. Ishida, Mater. Sci. Eng. 438, 1054 (2006)

8. P.J. Brown, K. Ishida, R. Kainuma, T. Kanomata, K.U. Neumann, K. Oikawa, B. Ouladdiaf, K.R.A. Ziebeck, J. Phys.: Condens. Matter 17, 1301 (2005)

9. V.A. Chernenko, J. Pons, E. Cesari, I.K. Zasimchuk, Scripta Materialia 50, 225 (2004)

10. K. Oikawa, T. Ots, F. Gejima, T. Ohmori, R. Kainuma, K. Ishida, Mater. Trans. 42, 2472 (2001)

11. K. Oikawa, L. Wulff, T. Iijima, F. Gelima, T. Ohmori, A. Fujita, K. Fukamichi, R. Kainuma, K. Ishida, Appl. Phys. Lett. 79, 3290 (2001)

12. M. Wuttig, J. Li, C. Cracuinescu, Scripta Materialia 44, 2393 (2002)

13. C. Cracuinescu, Y. Kishi, A.T. Lograsso, M. Wuttig, Scripta Materialia 47, 285 (2002)

14. K. Oikawa, T. Ota, Y. Tanaka, T. Omori, R. Kainuma, K. Ishida, Trans. Mater. Res. Soc. Jpn. 28, 265 (2003)

15. N. Koeda, Y. Sutou, T. Omori, K. Oikawa, R. Kainuma, K. Ishida, Scripta Materialia 52, 1153 (2005)

16. K. Oikawa, N. Koeda, Y. Sutou, T. Omori, R. Kainuma, K. Ishida, Mater. Trans. 45, 2780 (2004)

17. H. Morito, A. Fujita, K. Oikawa, K. Fukamichi, R. Kainuma, T. Kanomata, K. Ishida, J. Phys.: Condens. Matter 21, 076001 (2009)

18. Y.I.T. Omori, K. Oikawa, Y. Sutou, R. Kainuma, K. Ishida, Mater. Sci. Eng. 438, 970 (2006)

19. T. Takayama, S. Shinohara, K. Ishida, T. Nishizawa, J. Phase Equilibria 16, 390 (1995)

20. J.V. Humbeeck, J. Janssen, N. Mwamba, L. Delaey, Scripta Metall. 18, 893 (1984)

21. J. Gil, J.M. Guilemany, J. Mater. Sci. Lett. 11, 493 (1992)

22. H. Numakura, M. Ishimoto, M. Wuttig, Mater. Sci. Eng. A 442, 195 (2006)

23. Z. Lei, J. Cheng-Bao, S. Jia-Xiang, X. Hui-Bin, Chinese Physics B 18, 1647 (2009)

24. N.K. Jaggi, K.R.P.M. Rao, A.K. Grover, L.C. Gupta, R. Vijayaraghavan, L.D. Khoi, Hyperfine Interactions 4, $402(1978)$

25. G. Kresse, J. Furthmüller, Phys. Rev. B 54, 11169 (1996) 
26. G. Kresse, D. Joubert, Phys. Rev. B 59, 1758 (1999)

27. J.P. Perdew, K. Burke, M. Ernzerhof, Phys. Rev. Lett. 77, 3865 (1996)

28. H. Ebert, in Lecture Notes in Physics, edited by Springer (H. Dreysse, Berlin, 2000), Vol. 50, p. 191

29. Ebert et al, http://olymp.cup.uni-muenchen.de/ak/ebert/SPRKKR 